\title{
IKKe deficiency inhibits acute lung injury following renal ischemia reperfusion injury
}

\author{
CHAO MENG ${ }^{1,2}$, YI QIAN ${ }^{3}$, CUI ZHANG ${ }^{1}$, HAN LIU $^{1}$, XINWEI MU ${ }^{1}$ and AIPING ZHANG ${ }^{2}$ \\ Departments of ${ }^{1}$ Critical Care Medicine and ${ }^{2}$ Cardiothoracic Surgery, Nanjing First Hospital, Nanjing Medical University, \\ Nanjing, Jiangsu 210001; ${ }^{3}$ State Key Laboratory of Reproductive Medicine, Clinical Center of Reproductive Medicine, \\ The First Affiliated Hospital of Nanjing Medical University, Nanjing, Jiangsu 210009, P.R. China
}

Received January 14, 2020; Accepted September 1, 2020

DOI: $10.3892 / \mathrm{mmr} .2020 .11532$

\begin{abstract}
Renal ischemia reperfusion injury (IRI) after surgery may promote acute lung injury (ALI) by inducing an inflammatory response. However, the underlying molecular mechanism is still unclear. Studies have reported that inhibitor of $\kappa \mathrm{B}$ kinase $(\mathrm{IKK}) \varepsilon$ primarily regulates inflammation and cell proliferation. The present study aimed to investigate the regulatory role of IKKe in ALI in mice, in order to provide an experimental basis for preventing ALI following surgery-induced renal IRI. C57BL/6J wild-type (WT) and IKK $\varepsilon$ knockout $\left(\mathrm{IKK}^{--}\right)$mice underwent bilateral renal pedicle occlusion. The plasma creatinine concentration, urea nitrogen level and lung wet-to-dry ratio were measured at baseline, and at 24 and $48 \mathrm{~h}$ after declamping. The histological localization and protein levels of inflammatory factors, such as tumor necrosis factor (TNF)- $\alpha$, interleukin (IL)- $1 \beta$ and IL-10, were analyzed in lung tissues. Subsequently, the interactions between IKKe and components of the nuclear factor (NF)- $\mathrm{kB}$ pathway were studied. The results of the present study demonstrated that the $\mathrm{IKK}^{-/}$groups displayed similar renal function but less pulmonary edema compared with that of the WT groups. The levels of proinflammatory factors in the lungs were significantly upregulated in WT mice compared with those in IKK $\varepsilon^{-/-}$mice after IRI surgery. The NF- $\kappa B$ pathway components and downstream factors were substantially upregulated in the WT groups after acute ischemic kidney injury, and these effects were significantly inhibited in the $\mathrm{IKK \varepsilon}^{-1-}$ groups. Based on these data, the present study hypothesized
\end{abstract}

Correspondence to: Dr Xinwei Mu, Department of Critical Care Medicine, Nanjing First Hospital, Nanjing Medical University, 68 Changle Road, Nanjing, Jiangsu 210001, P.R. China

E-mail: muxinwei1964@yahoo.com

Dr Aiping Zhang, Department of Cardiothoracic Surgery, Nanjing First Hospital, Nanjing Medical University, 68 Changle Road, Nanjing, Jiangsu 210001, P.R. China

E-mail: zhangaiping876@126.com

Key words: inhibitor of $\kappa \mathrm{B}$ kinase $\varepsilon$, renal ischemia reperfusion injury, acute lung injury, nuclear factor- $\kappa \mathrm{B}$, inflammation that IKKe may serve a negative role in kidney-lung crosstalk after renal IRI and may be a novel target for the treatment of patients with renal IRI.

\section{Introduction}

Acute kidney injury (AKI) is a frequent complication after cardiac surgery and abdominal aortic aneurysm repair $(1,2)$. However, $\sim 50 \%$ of AKI cases in hospitalized patients are caused by renal ischemia reperfusion injury (IRI) (3). The pathophysiology of AKI is very complex and combines major ischemia-induced cell stress, a significant burst of free radicals and pro-inflammatory cytokines [such as interleukin (IL)-1 $\beta$, IL-6 and tumor necrosis factor (TNF)- $\alpha$ )] evoking a pro-inflammatory cascade, and subsequent injuries on distant organs, including the lung, heart, liver and brain (4-6). This organ crosstalk phenomenon is well-known in critical care medicine as multiple organ failure due to systemic inflammatory response syndrome. Acute lung injury (ALI) is the most clinically relevant remote organ dysfunction associated with AKI (5). The pathological characteristics of ALI are increased pulmonary vascular permeability, lung edema and alveolar hemorrhage (7-9). A previous study indicated that when both AKI and ALI occur, the overall morbidity is as high as $80 \%$ (4). Therefore, improved understanding of the effects of renal IRI on remote organs, especially on the lungs, is urgently needed.

Nuclear factor (NF) $-\kappa \mathrm{B}$ proteins are a family of ubiquitously expressed transcription factors. In their inactive forms, $N F-\kappa B$ proteins are bound by members of the inhibitor of $\kappa \mathrm{B}(\mathrm{I} \kappa \mathrm{B})$ family. I $\kappa \mathrm{B}$ kinase $(\mathrm{IKK}) \varepsilon$ is a member of the IKK family, which influences $N F-\kappa B$ signaling and concomitant gene expression downstream of $\mathrm{I} \kappa \mathrm{B}(10,11)$. A previous study indicated that the $\mathrm{NF}-\kappa \mathrm{B}$ pathway may aggravate tubular injury and exacerbate a maladaptive inflammatory response in renal ischemia reperfusion-induced AKI (12). IKK family members may be therapeutic targets for lung injury, and the beneficial effects of IKK proteins may be mediated by inhibition of the NF- $\kappa$ B pathway (13).

According to Park et al (14) and Bulek et al (15), IKKe may serve an important role in enhancing lipopolysaccharide-induced and IL-17-mediated inflammatory responses, including increasing the transcription of inflammation-related genes in primary airway epithelial cells, and exacerbating the 
severity of neutrophilia and pulmonary inflammation in ALI. However, the molecular mechanism of ALI in the setting of renal IRI remains unclear. Based on the results of a previous study, NF- $\kappa \mathrm{B}$-dependent gene expression induced by TNF- $\alpha$ or IL-1 may be abrogated by IKKe (16); however, to the best of our knowledge, reports of the role of IKKe in kidney-lung crosstalk in renal IRI are lacking.

The present study aimed to investigate the possible molecular mechanisms by which IRI induces ALI, and the effect of the NF- $\kappa \mathrm{B}$ pathway on kidney-lung crosstalk in renal IRI by primarily focusing on the role of IKKe. The present study may improve understanding of the pathological mechanisms underlying IRI-induced ALI and the significance of anti-inflammatory treatments for patients with IRI.

\section{Materials and methods}

Animals. IKKe knockout C57BL/6J mice (IKKe $\varepsilon^{-/}$) (male; age, 6-8-weeks; weight, 25-30 g) were purchased from the Jackson Laboratory and housed in the Model Animal Research Center of Nanjing University (Nanjing, China). Wild-type (WT) C57BL/6J mice (male; age, 6-8-weeks; weight, 25-30 g) were obtained from the Animal Research Center of Nanjing Medical University (Nanjing, China). All mice were housed at 2 animals/cage in a light-controlled environment at $24 \pm 1^{\circ} \mathrm{C}$, 40-80\% humidity, 12-h light/dark cycles with access to food and water ad libitum throughout the experimental period. Animal experiments were performed in compliance with the Institute of Laboratory Animal Research Guide for the Care and Use of Laboratory Animals of the NIH and approved by the Institutional Animal Care and Use Committee of Nanjing Medical University.

Surgical procedures. A total of five experimental groups were evaluated in this study ( $n=6$ in each group): Two IRI groups (WT and $\mathrm{IKK} \varepsilon^{-/}$), two sham groups (WT and IKKe $\varepsilon^{-/-}$) and one control group (WT; no surgery). All procedures were performed using strict sterile techniques after inducing anesthesia with an intraperitoneal (IP) injection of pentobarbital (50 mg/kg body weight). Adequate anesthesia was assessed by pinching the paw and tail. Animals from each group were placed on a heating blanket prior to sham and IRI operations. Renal IRI was performed as previously described (17). Briefly, the back of the mouse was shaved and a $1.5-\mathrm{cm}$ incision was made on both sides. Two vascular clamps were applied across both renal pedicles for $45 \mathrm{~min}$. Occlusion was visually verified by monitoring the change in the color of the kidney to a paler hue. After clamp removal, the restoration of blood flow to the kidney was confirmed by the return of the original color. The incisions on the back of each mouse were closed in two layers with sutures, and mice were returned to their cages for 24 or $48 \mathrm{~h}$. The animals were allowed to recover, and had free access to food and water. Animals in the sham groups underwent an identical procedure without vascular clamp placement. Blood and tissue samples were separately collected at different time points ( 24 and $48 \mathrm{~h}$ ) from different groups.

Plasma parameters. At different time points after operation (24 and $48 \mathrm{~h}$ ), mice were anesthetized with an IP injection of pentobarbital (50 $\mathrm{mg} / \mathrm{kg}$ body weight), and the adequacy of anesthesia was evaluated by monitoring hind limb reflexes. Blood samples $(\sim 0.5 \mathrm{ml})$ were obtained from the retroorbital plexus and centrifuged at $1,509 \times \mathrm{g}$ for $15 \mathrm{~min}$ at $4^{\circ} \mathrm{C}$ to obtain serum. The samples were stored at $-80^{\circ} \mathrm{C}$ until further use. Serum creatinine (SCr) and blood urea nitrogen (BUN) levels were measured as renal function markers using an Olympus AU2700 automatic biochemistry apparatus (Olympus Corporation).

Tissue collection and lung wet-to-dry ratio. Immediately after the blood samples collection procedure, mice were euthanized with an overdose of pentobarbital ( $150 \mathrm{mg} / \mathrm{kg}$ body weight). The inferior lobe of the left lung was harvested to measure the lung wet-to-dry ratio. First, an arteriovenous (AV) fistula needle was placed in the left atrium. Saline was slowly forced through another AV fistula needle inserted into the right ventricle using the injector until the lung changed color from red to white. The upper lobe of the left lung was harvested and preserved in liquid nitrogen for western blotting. The left main bronchus was isolated and cross-clamped. The right lung was filled with $0.5 \%$ low-melting point agarose in $10 \%$ formalin at a constant pressure of $25 \mathrm{~cm} \mathrm{H}_{2} \mathrm{O}$ through a tracheotomy with an AV fistula needle, allowing the homogenous expansion of the lung parenchyma. The lung wet-to-dry ratio was measured by desiccating the lung at $80^{\circ} \mathrm{C}$ until a constant weight was obtained. The ratio was calculated as an indicator of lung edema.

Histological analysis using hematoxylin and eosin (H\&E) staining. For histopathological evaluation of lung injury, lung tissues were obtained from the mice and subsequently fixed in $10 \%$ formaldehyde at room temperature for $24 \mathrm{~h}$ and embedded in paraffin. The $5-\mu \mathrm{m}$ sections were heated at $60^{\circ} \mathrm{C}$ for $1 \mathrm{~h}$, before being dewaxed in xylene and rehydrated using a descending ethanol series. H\&E staining was then performed on sections, with hematoxylin for $10 \mathrm{~min}$ room temperature and eosin for $5 \mathrm{~min}$ at room temperature. Stained sections were visualized using a light microscope (magnification, $\mathrm{x} 200$ ) by a pathologist in a blinded manner.

Western blot analysis. IHC and western blotting were performed as previously described $(12,17)$. Lung tissue samples were ground in liquid nitrogen and lysed using RIPA lysis buffer (Beyotime Institute of Biotechnology) for $30 \mathrm{~min}$. Total protein was quantified using a bicinchoninic acid assay and $50 \mu \mathrm{g}$ protein was separated using 12\% SDS-PAGE for $90 \mathrm{~min}$. The separated proteins were subsequently transferred onto polyvinylidene difluoride membranes and blocked in TBS with 5\% skimmed milk for $2 \mathrm{~h}$ at room temperature. The membranes were incubated with primary antibodies against GAPDH (1:1,000; cat. no. 5174; Cell Signaling Technology, Inc.), IKKe (1:500; cat. no. ab124766; Abcam), NF-кB phosphorylated p50 (pi-p50; 1:200; cat. no. sc-271908; Santa Cruz Biotechnology, Inc.), NF- $\kappa$ B phosphorylated p65 (pi-p65; 1:200; cat. no. sc-166748; Santa Cruz Biotechnology, Inc.),

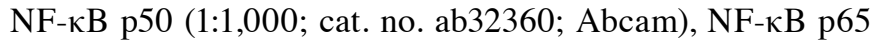
(1:800; cat. no. ab16502; Abcam) overnight at $4^{\circ} \mathrm{C}$. Following the primary antibody incubation, the membranes were subsequently incubated with a horseradish peroxidase-conjugated secondary antibody (cat. no. sc-2370; 1:5,000; Santa Cruz 

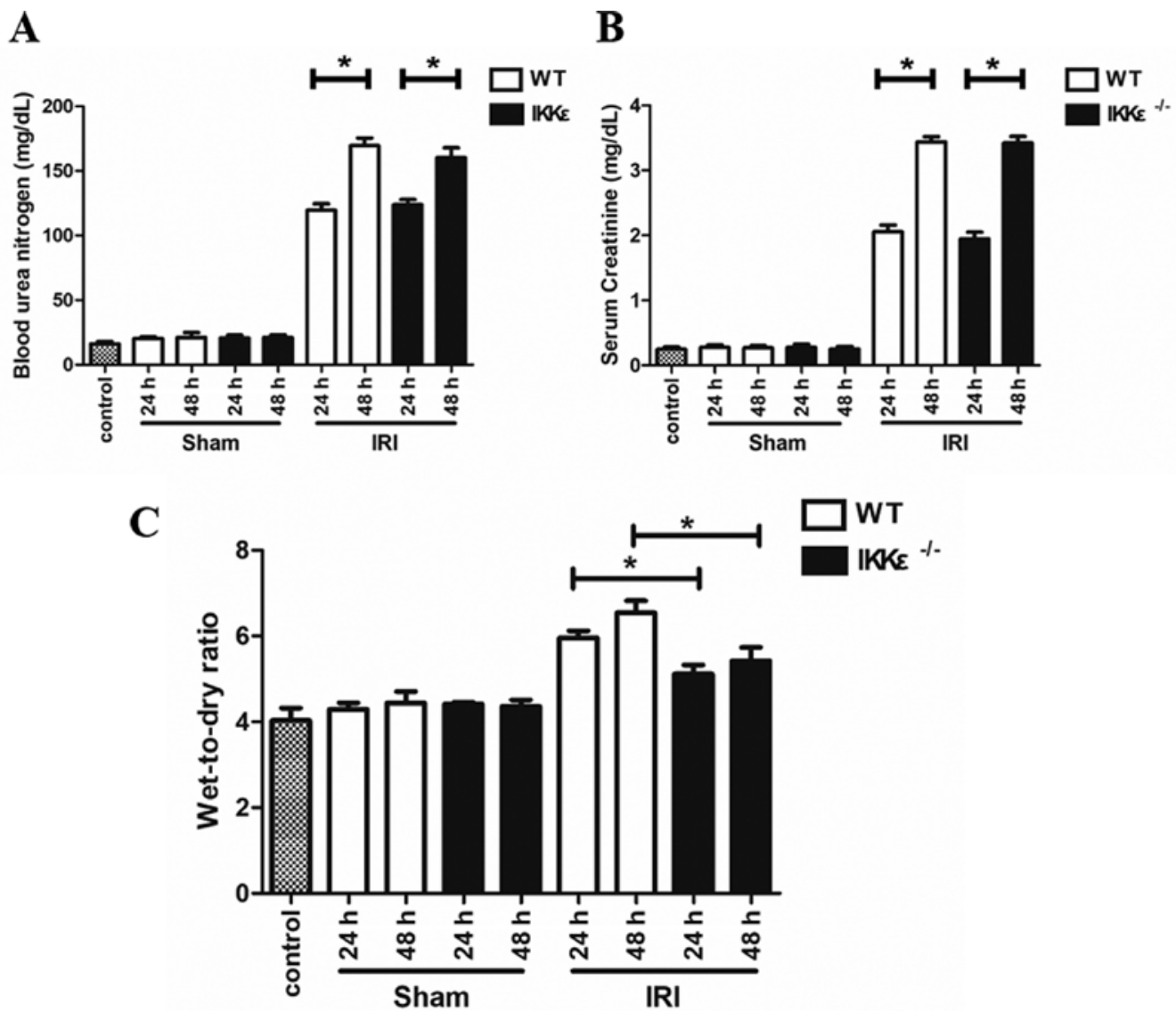

Figure 1. Changes in renal function and the lung wet-to-dry ratio following experimental ALI. (A) Blood urea nitrogen and (B) serum creatinine levels. (C) Lung wet-to-dry ratio was measured to evaluate pulmonary function and edema. "P<0.05. IRI, ischemia reperfusion injury; WT, wild-type; IKK ${ }^{-/}$, inhibitor of $\kappa \mathrm{B}$ kinase $\varepsilon$ knockout.

Biotechnology Inc.) for $1 \mathrm{~h}$ at $37^{\circ} \mathrm{C}$. The protein-antibody complexes were visualized using Pierce ${ }^{\mathrm{TM}}$ Fast Western Blot Kit, ECL Substrate (cat. no. 35050; Thermo Fisher Scientific, Inc.) with a chemiluminescence instrument (Tanon Science and Technology Co., Ltd.). Protein expression was quantified using ImagePro Plus software (version 6.0; Media Cybernetics, Inc.).

Immunohistochemical analysis. Immunohistochemistry was performed using paraffin-embedded tissue sections cut at $4-\mu \mathrm{m}$ thickness mounted on glass slides. The slides were then deparaffinized and rehydrated. Then, the lung sections were incubated with primary antibodies against TNF- $\alpha$ (1:200; cat. no. ab9739; Abcam), Ki67 (1:2,000; cat. no. ab15580; Abcam), IL-1 $\beta$ (1:200; cat. no. sc-7884; Santa Cruz Biotechnology, Inc.), and IL-10 (1:400; cat. no. bs-0698R; BIOSS), NF- $\kappa$ B phosphorylated p50 (pi-p50; 1:200; cat. no. sc-271908; Santa Cruz Biotechnology, Inc.), NF-кB phosphorylated p65 (pi-p65; 1:200; cat. no. sc-166748; Santa Cruz Biotechnology, Inc.) and then with biotin secondary antibodies (B3640; Sigma-Aldrich; Merck KGaA) at room temperature for $30 \mathrm{~min}$.

The IHC score was determined using the Fromowitz standard as previously described (18). The percentage of positive stained cells was graded as follows, $0-5 \%, 0 ; 6-25 \%, 1$; $26-50 \%, 2 ; 51-75 \%, 3 ;>75 \%, 4$. The intensity of staining was graded as follows: Absent or faint blush, 0; weak, 1; moderate, 2 ; strong, 3 . Then the two scores were added.
Statistical analysis. The data are presented as the mean \pm standard error of the mean of at least three independent repeats. The results were analyzed by one-way ANOVA followed by a Tukey's post hoc test. The IHC scores were compared by Mann-Whitney U test. SPSS 17 software (SPPS, Inc.) was used to perform the statistical analysis. $\mathrm{P}<0.05$ was considered to indicate a statistically significant difference.

\section{Results}

Renal function significantly decreases following experimental renal IRI. SCr and BUN levels were measured in $\mathrm{IKK}^{-/-}$and WT mice at 24 and $48 \mathrm{~h}$ after surgical IRI to confirm the decreased renal function. Compared with the control and sham groups, the IRI group mice exhibited significant increases in $\mathrm{SCr}$ values and BUN levels at 24 and $48 \mathrm{~h} \quad(\mathrm{P}<0.05$; Fig. 1A and B). Additionally, no significant difference was observed in renal function between the WT and $\mathrm{IKK}^{-/-}$groups 24 and $48 \mathrm{~h}$ after surgical IRI ( $\mathrm{P}>0.05$; Fig. $1 \mathrm{~A}$ and $\mathrm{B})$.

$I K K^{-/-}$mice exhibit significantly weaker acute disease and pulmonary edema compared with that of WT mice. The lung wet-to-dry ratio was detected to evaluate the degree of pulmonary edema. The results demonstrated a gradually increasing trend after surgical IRI (Fig. 1C). In addition, the IKK $\varepsilon^{-/}$mice group exhibited significantly weaker pulmonary edema compared with that of the WT mice in 24 and $48 \mathrm{~h}(\mathrm{P}<0.05)$. 


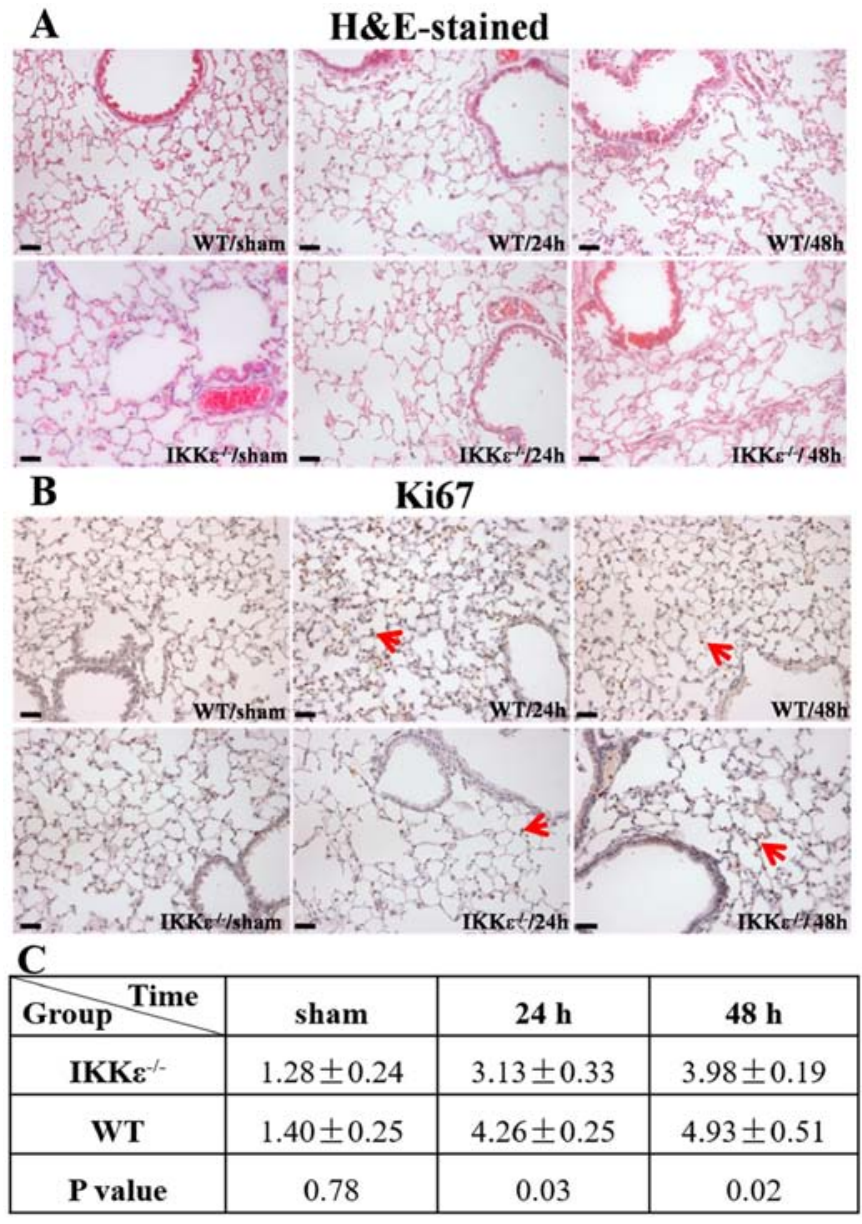

Figure 2. H\&E staining and Ki67 IHC. (A) Representative images of $\mathrm{H} \& \mathrm{E}$-stained lung tissues from the IKKe ${ }^{-/}$and WT groups at 24 or $48 \mathrm{~h}$ following IRI. (B) IHC of proliferation-associated antigen Ki67 in lung tissues from the IKK $\varepsilon^{-1}$ and WT groups at 24 or $48 \mathrm{~h}$ following IRI. (C) IHC scores of IKK $\varepsilon^{-/}$and WT IRI groups. Scale bar, $50 \mu \mathrm{m}$. Arrows indicate positive cells. IHC, immunohistochemistry; H\&E, hematoxylin and eosin; IRI, ischemia reperfusion injury; WT, wil-type; IKK $\varepsilon^{-1}$, inhibitor of $\kappa \mathrm{B}$ kinase $\varepsilon$ knockout.

IKKE knockout attenuates experimental renal IRI-induced lung inflammation. H\&E-stained lung sections were examined to further determine whether IKKe knockout affects experimental renal IRI-induced ALI. Since the present study indicated that there was no significant difference in renal function and pulmonary edema between the control and sham groups at 24 and $48 \mathrm{~h}$, a 48 -h timepoint was used for the sham group in subsequent experiments. The results demonstrated that in the WT group, renal IRI induced persistent interstitial edema, focal alveolar hemorrhage, alveolar wall thickening and inflammatory cell infiltration. By contrast, lung tissues from the $\mathrm{IKK}^{-1-}$ group exhibited less damage, which manifested with a disordered and uneven distribution (Fig. 2A).

Immunohistochemical analysis of the proliferation-associated antigen Ki67 (Fig. 2B) indicated the absence of mitotic figures, and very few cells in the sham group were Ki67-positive. By contrast, compared with those in the IKK $\varepsilon^{-1-}$ groups, the number of Ki67-positive cells was significantly increased in the WT groups at 24 and 48 h (Fig. 2B and C); thus suggesting cellular proliferation occurred following kidney IRI in the WT group.
Inflammatory markers, including TNF- $\alpha$, IL-1 $\beta$ and IL-10, were detected in lung tissues. The results demonstrated that the expression levels of TNF- $\alpha$, IL- $1 \beta$ and IL-10 were significantly lower in the $\mathrm{IKK}^{-/}$groups compared with those in the WT groups 24 and $48 \mathrm{~h}$ post-surgery $(\mathrm{P}<0.05$; Fig. 3A-D). These results suggested that inflammatory activity was suppressed in the lungs of $\mathrm{IKK}^{-/-}$mice.

IKKE ablation blocks NF- $\kappa B$ activation induced by acute ischemic kidney injury. Studies have demonstrated that IKKe participates in renal IRI (12); however, the exact mechanism is still unclear. Therefore, the levels of downstream factors of the NF- $\mathrm{KB}$ pathway, such as pi-p50 and pi-p65, in lung tissues was examined by immunohistochemical staining. The results demonstrated that the expression levels of pi-p50 and pi-p65 were significantly lower in bronchial epithelial cells of the IKKe $\varepsilon^{-/}$groups compared with those in the WT groups ( $\mathrm{P}<0.05$; Fig. 4A-C).

Western blotting was performed to determine the expression levels of pi-p50 and pi-p65, which are the functionally active and nuclear forms of NF- $\mathrm{KB}$ (11). The results demonstrated that IKKe expression levels gradually increased in the WT group after acute ischemic kidney injury (Fig. 5). In addition, the expression levels of pi-p50 and pi-p65 were significantly reduced in the $\mathrm{IKK}^{-/}$group compared with those in the WT group $(\mathrm{P}<0.05)$, whereas no significant differences were observed in the total $\mathrm{p} 50$ and $\mathrm{p} 65$ protein expression levels (Fig. 5).

\section{Discussion}

The pathogenesis of renal IRI is complex and is still not entirely understood. However, inflammation is currently accepted as an important pathogenic component (19). Renal IRI has been reported to result in endothelial and leukocyte activation, reactive oxygen species production, tubular cell death and the release of inflammatory mediators, such as cytokines and chemokines (19). AKI has been reported to activate host innate and adaptive immune responses, and experimental data have identified both soluble and cellular mediators activated by the post-ischemic kidney that drive ALI $(5,20,21)$. Despite the clinical association between renal IRI and ALI, little is known about the molecular mechanism of kidney-lung crosstalk following renal IRI. A previous study has observed that increased expression of TNF- $\alpha$ in the lungs may induce pulmonary inflammatory damage (22). The present study used an IKK $\varepsilon^{-/-}$mouse model of unilateral IRI and investigated the possible roles of $\mathrm{IKK}^{-/}$and related inflammatory mediators, such as TNF $\alpha$, IL-10 and IL-1 $\beta$. To the best of our knowledge, the present study is the first to demonstrate that the IKKe pathway may serve a role in kidney-lung crosstalk following renal IRI.

The results of the present study demonstrated the $\mathrm{IKK}^{-1-}$ and WT groups exhibited similar decreased renal function following experimental AKI; however, the lung wet-to-dry ratio was significantly decreased in the $\mathrm{IKK \varepsilon}^{-/}$group compared with in the WT group. In addition, this study examined the morphological and molecular alterations in lung tissues to investigate the effect of IKK $\varepsilon$ on ALI following renal IRI. A series of histopathological changes were demonstrated 
A

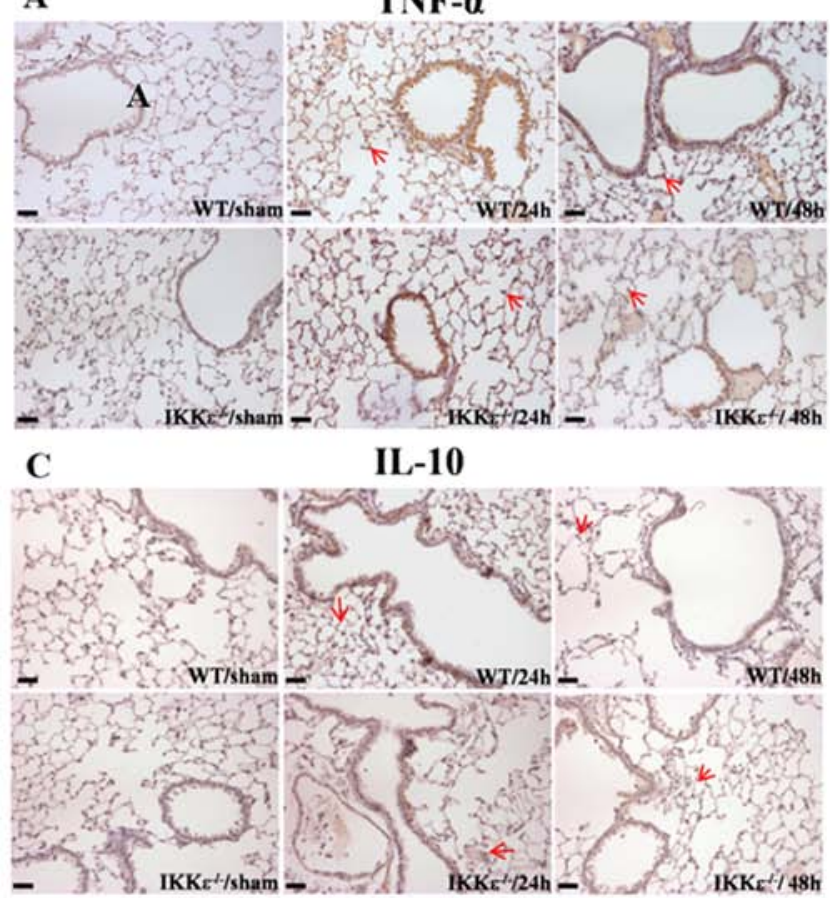

B

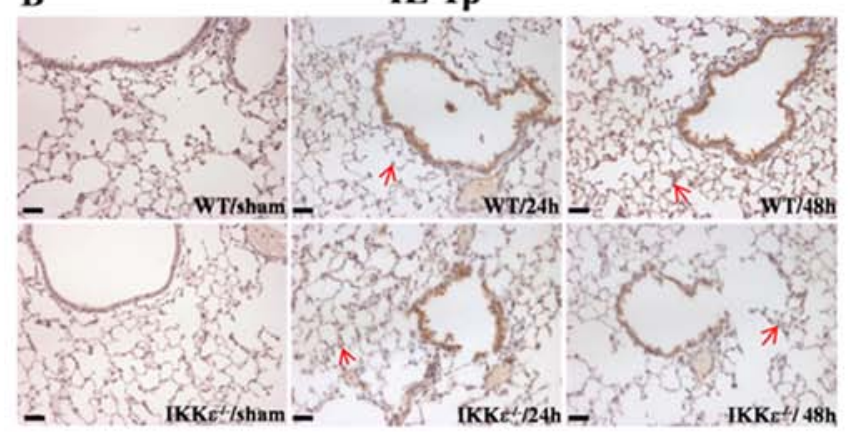

D

\begin{tabular}{|c|c|c|c|c|c|c|c|c|c|}
\hline Time & & TNF- $a$ & & & IL-1 $1 \beta$ & & & IL -10 & \\
\hline Group & sham & $24 \mathrm{~h}$ & $48 \mathrm{~h}$ & sham & $24 \mathrm{~h}$ & $48 \mathrm{~h}$ & sham & $24 \mathrm{~h}$ & $48 \mathrm{~h}$ \\
\hline IKKe $\boldsymbol{c}^{+}$ & $\begin{array}{c}1.22 \pm \\
0.23\end{array}$ & $\begin{array}{c}2.50 \pm \\
0.27\end{array}$ & $\begin{array}{c}3.40 \pm \\
0.36\end{array}$ & $\begin{array}{c}1.10 \pm \\
0.32\end{array}$ & $\begin{array}{c}1.52 \pm \\
0.61\end{array}$ & $\begin{array}{c}3.62 \pm \\
0.21\end{array}$ & $\begin{array}{c}0.98 \pm \\
0.34\end{array}$ & $\begin{array}{c}1.34 \pm \\
0.35\end{array}$ & $\begin{array}{c}2.70 \pm \\
0.37\end{array}$ \\
\hline WT & $\begin{array}{c}1.38 \pm \\
0.50\end{array}$ & $\begin{array}{c}4.63 \pm \\
0.32\end{array}$ & $\begin{array}{c}5.53 \pm \\
0.42\end{array}$ & $\begin{array}{l}1.21 \pm \\
0.28\end{array}$ & $\begin{array}{c}3.57 \pm \\
0.25\end{array}$ & $\begin{array}{c}5.13 \pm \\
0.14\end{array}$ & $\begin{array}{c}1.02 \pm \\
0.32\end{array}$ & $\begin{array}{c}3.14 \pm \\
0.20\end{array}$ & $\begin{array}{c}5.23 \pm \\
0.25\end{array}$ \\
\hline$P$ value & 0.12 & $<0.001$ & $<0.001$ & 0.53 & 0.02 & $<0.001$ & 0.56 & 0.01 & $<0.001$ \\
\hline
\end{tabular}

Figure 3. IHC of inflammatory factors and downstream factors of the nuclear factor- $\kappa$ B pathway in lung tissues. Representative images of IHC for (A) TNF- $\alpha$, (B) IL-1 $\beta$ and (C) IL-10 in lung tissues from the IKKe ${ }^{-/}$and WT groups at 24 or $48 \mathrm{~h}$ following IRI. (D) IHC scores of IKK $\varepsilon^{-/}$and WT IRI groups. Scale bar, $50 \mu \mathrm{m}$. Arrows indicate positive cells. IHC, immunohistochemistry; IRI, ischemia reperfusion injury; WT, wild-type; IKK $\varepsilon^{-/}$, inhibitor of $\kappa \mathrm{B}$ kinase $\varepsilon$ knockout; TNF, tumor necrosis factor; IL, interleukin.

A
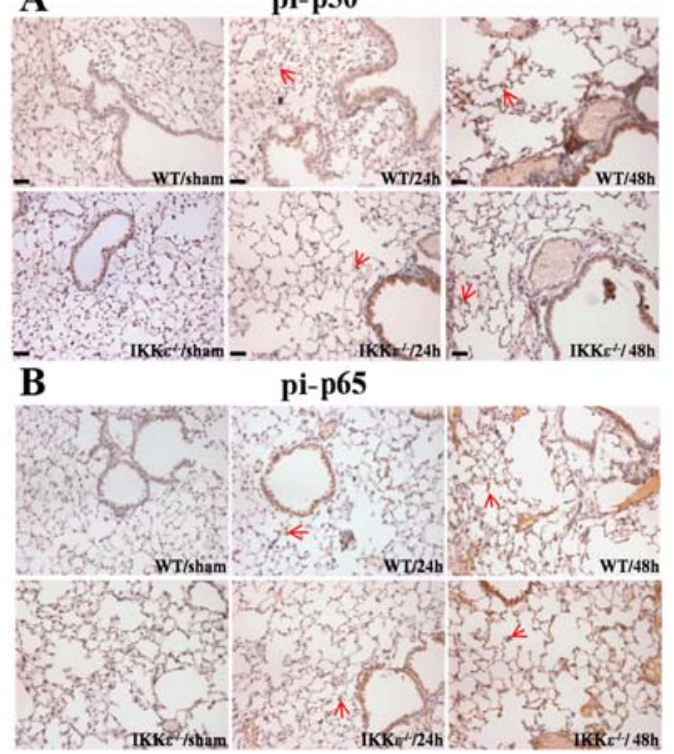

C

\begin{tabular}{|c|c|c|c|}
\hline Group Time & sham & $24 \mathrm{~h}$ & $48 \mathrm{~h}$ \\
\hline IKK $\varepsilon^{-/ *}$ & $1.23 \pm 0.24$ & $3.24 \pm 0.13$ & $4.32 \pm 0.24$ \\
\hline WT & $1.38 \pm 0.44$ & $4.28 \pm 0.22$ & $5.41 \pm 0.30$ \\
\hline Pvalue & 0.08 & 0.03 & 0.02 \\
\hline
\end{tabular}

Figure 4. IHC of downstream factors of the NF- $\mathrm{B}$ pathway in lung tissues. Representative images of IHC staining for (A) pi-p50 and (B) pi-p65 in lung tissues from the IKKe $\varepsilon^{-/-}$and WT groups at 24 or $48 \mathrm{~h}$ following IRI. (C) IHC scores of IKK $\varepsilon^{-/}$and WT IRI groups. Scale bar, $50 \mu \mathrm{m}$. Arrows indicate positive cells. NF- $\kappa \mathrm{B}$, nuclear factor- $\kappa \mathrm{B}$; IRI, ischemia reperfusion injury; IHC, immunohistochemistry; WT, wild-type; IKK $\varepsilon^{-/}$, inhibitor of $\kappa \mathrm{B}$ kinase $\varepsilon$ knockout; pi, phosphorylated. by H\&E staining; renal IRI induced persistent interstitial edema, focal alveolar hemorrhage, alveolar wall thickening and inflammatory cell infiltration in the WT group, whereas lung tissues from the $\mathrm{IKK} \varepsilon^{-/}$group exhibited less damage. The present findings demonstrated that IKKe knockout may reduce lung edema after renal IRI. Thus, it was hypothesized that IKKع deficiency may contribute to the reduction of inflammation in the lungs after renal IRI. Subsequently, Ki67 levels were measured in lung tissues by IHC to explore cell proliferation following the pathological changes. The number of Ki67-positive cells was greater in the lungs of the WT group compared with that in the IKK $\varepsilon^{-/-}$group. Thus, lung cell proliferation was significantly reduced after inflammatory injury in the absence of IKKe. Thus, it was hypothesized that IKK $\varepsilon$ may be associated with inflammatory cell infiltration and lung tissue destruction.

In the present study, the expression of inflammatory markers (TNF $\alpha$, IL-10, and IL-1 $\beta$ ) was detected in lung tissues. The results demonstrated that TNF $\alpha$ expression levels were elevated after renal IRI treatment, but were inhibited by IKK $\varepsilon$ knockout. Higher TNF- $\alpha$ expression levels were observed in the airway epithelial cells of the WT group after renal IRI treatment, whereas lower expression levels were observed in the $\mathrm{IKK \varepsilon}^{-/}$group. Renal IRI has been reported to activate soluble $\mathrm{TNF} \alpha$, and thus induce $\mathrm{TNF} \alpha$ receptor 1-dependent pulmonary cell apoptosis and microvascular barrier dysfunction (22). Thus, TNF- $\alpha$ may serve a key role in aggravating the downstream effects of renal IRI. IL-1 $\beta$ is an important protein participating in NF- $\kappa \mathrm{B}$-induced inflammatory responses, which has been shown to exhibit a biphasic distribution in IRI injury models $(23,24)$. Previous studies 


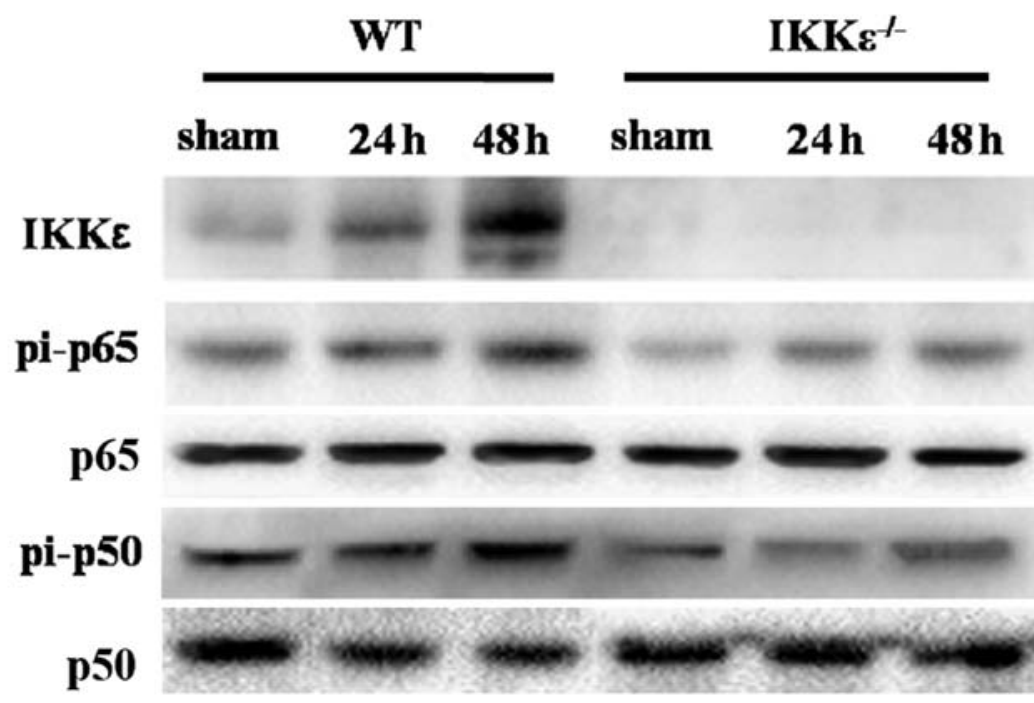

\section{GAPDH}

B
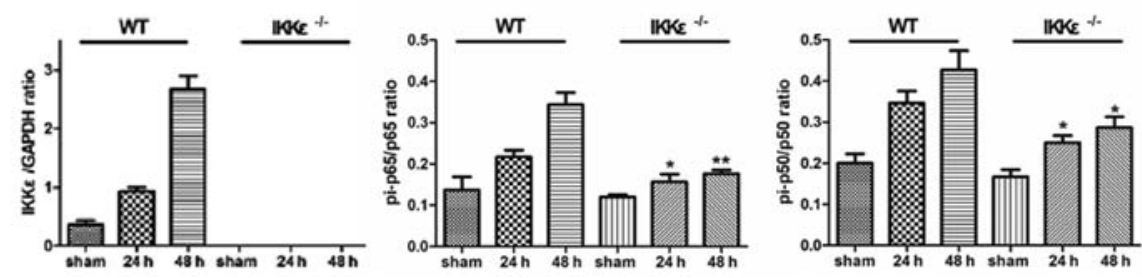

Figure 5. Differential expression of pi-p50 and pi-p65 in lung tissues from the IKK $\varepsilon^{-/}$and WT groups at 24 or $48 \mathrm{~h}$ following IRI. (A) Representative western blotting results of each group. (B) Relative protein expression levels were normalized to GAPDH/total protein expression ( $\mathrm{n}=4$ ). ${ }^{*} \mathrm{P}<0.5$, ${ }^{* *} \mathrm{P}<0.01 \mathrm{vs}$. WT group.

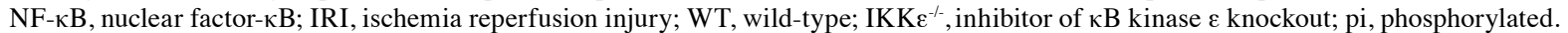

observed that IL-1 $\beta$ expression levels were significantly increased and remained high during the reperfusion period compared with controls (23-25). In the present study, the expression levels of IL-1 $\beta$ were investigated during the reperfusion period and the results indicated that IL-1 $\beta$ expression levels were higher in the WT groups compared with those in the IKK $\varepsilon^{-/-}$groups. These results suggested that IL-1 $\beta$ may be related to IKKe activation. According to previous studies, TNF- $\alpha$, IL-1 $\beta$, IL- 6 and other pro-inflammatory cytokines inhibited the anti-inflammatory effects mediated by IL-10, indicating that these factors may perform similar roles in IRI-related pathways $(26,27)$. Consistent with other studies, the results of the present study demonstrated that the expression levels of IL-10 were higher in the WT groups compared with those in $\mathrm{IKK}^{-/}$groups. Thus, it was concluded that the levels of inflammatory and anti-inflammatory factors were significantly increased in lung tissues following renal IRI, indicating an inflammation reaction, and the effects were inhibited by IKK $\varepsilon$ knockout. Moreover, in addition to anti-inflammatory effects, IL-10 could also inhibit superoxide production and reduce metalloproteinase release. The present findings may result from a compensatory response of IL-10 to TNF- $\alpha$ and other pro-inflammatory cytokines.

Subsequently, the major components of the NF- $\kappa B$ signaling pathway were measured. Pi-p65/p50 activate downstream inflammatory factors of the NF- $\kappa$ B pathway, such as TNF $\alpha$, IL-10 and IL-1 $\beta(28,29)$. In the present study, the expression of the NF- $\mathrm{kB}$ cascade factors were investigated. The expression levels of pi-p65 and pi-p50 were demonstrated to be upregulated in the lungs of the WT groups following renal IRI compared with those of the IKK $\varepsilon^{-1}$ groups. IKK $\varepsilon$ phosphorylates NF- $\kappa B$ (pi-p65), contributing to the $\mathrm{NF}-\kappa \mathrm{B}-$ dependent expression of target genes in response to proinflammatory signals $(10,30,31)$. However, the inhibitory effects were not completely dependent on IKKe deficiency, indicating other signaling pathways may exist. Studies have observed that NF- $\mathrm{KB}$ activation is separately mediated by myeloid differentiation primary response 88 via IKK $\alpha / \beta$ and by TIR domain-containing adaptor-inducing interferon- $\beta$ via $\operatorname{IKK} \varepsilon(28,32,33)$.

Previous studies have reported that myeloperoxidase activity, neutrophil infiltration, blood oxygen saturation and pulmonary vascular permeability are important evaluation indicators of ALI $(5,20)$. Therefore, these factors could be further explored in future studies.

In conclusion, in the present study, a novel role for IKK $\varepsilon$ in regulating renal IRI-induced inflammation in mouse lungs was identified. In the absence of IKKe, the renal IRI-associated destruction of mouse lung tissue and inflammatory responses were substantially prevented, and the expression levels of components associated with $\mathrm{NF}-\mathrm{\kappa B}$ signaling were reduced. Based on these findings and 
previous studies suggesting a role for IKK $\varepsilon$ in NF- $\kappa \mathrm{B}$ activation, it was hypothesized that IKKe may serve a pivotal role in the activity of $\mathrm{NF}-\kappa \mathrm{B}$, which in turn could regulate the expression of genes involved in the inflammatory response in lungs following renal IRI.

\section{Acknowledgements}

Not applicable.

\section{Funding}

The current study was supported by a grant from the Science and Technology Development Fund of Nanjing Medical University (grant no. NMUB2019147).

\section{Availability of data and materials}

The datasets used and/or analyzed during the current study are available from the corresponding author on reasonable request.

\section{Authors' contributions}

CM designed the study and performed experiments. XM and AZ designed the study. CZ and HL performed experiments. YQ analyzed the data and wrote the manuscript. All authors read and approved the final manuscript.

\section{Ethics approval and consent to participate}

The present study was approved by the Institutional Animal Care and Use Committee of Nanjing Medical University.

\section{Patient consent for publication}

Not applicable.

\section{Competing interests}

The authors declare that they have no competing interests.

\section{References}

1. Brown JR, Cochran RP, Dacey LJ, Ross CS, Kunzelman KS, Dunton RF, Braxton JH, Charlesworth DC, Clough RA, Helm RE, et al: Perioperative increases in serum creatinine are predictive of increased 90-day mortality after coronary artery bypass graft surgery. Circulation 114 (1 Suppl): I409-I413, 2006.

2. Karkouti K, Wijeysundera DN, Yau TM, Callum JL, Cheng DC, Crowther M, Dupuis JY, Fremes SE, Kent B, Laflamme C, et al: Acute kidney injury after cardiac surgery: Focus on modifiable risk factors. Circulation 119: 495-502, 2009.

3. Star RA: Treatment of acute renal failure. Kidney Int 54: 1817-1831, 1998

4. Klein CL, Hoke TS, Fang WF, Altmann CJ, Douglas IS and Faubel S: Interleukin-6 mediates lung injury following ischemic acute kidney injury or bilateral nephrectomy. Kidney Int 74: 901-909, 2008

5. Awad AS, Rouse M, Huang L, Vergis AL, Reutershan J, Cathro HP, Linden J and Okusa MD: Compartmentalization of neutrophils in the kidney and lung following acute ischemic kidney injury. Kidney Int 75: 689-698, 2009.

6. Kinsey GR and Okusa MD: Pathogenesis of acute kidney injury: Foundation for clinical practice. Am J Kidney Dis 58: 291-301, 2011.
7. DengJ,HuX,YuenPSandStarRA:Alpha-melanocyte-stimulating hormone inhibits lung injury after renal ischemia/reperfusion. Am J Respir Crit Care Med 169: 749-756, 2004.

8. Kelly KJ: Distant effects of experimental renal ischemia/reperfusion injury. J Am Soc Nephrol 14: 1549-1558, 2003.

9. Nath KA, Grande JP, Croatt AJ, Frank E, Caplice NM, Hebbel RP and Katusic ZS: Transgenic sickle mice are markedly sensitive to renal ischemia-reperfusion injury. Am J Pathol 166: 963-972, 2005.

10. Clement JF, Meloche S and Servant MJ: The IKK-related kinases: From innate immunity to oncogenesis. Cell Res 18: 889-899, 2008

11. Vallabhapurapu S and Karin M: Regulation and function of NF-kappaB transcription factors in the immune system. Annu Rev Immunol 27: 693-733, 2009.

12. Marko L, Vigolo E, Hinze C, Park JK, Roel G, Balogh A, Choi M, Wubken A, Cording J, Blasig IE, et al: Tubular epithelial NF-KB activity regulates ischemic AKI. J Am Soc Nephrol 27: 2658-2669, 2016

13. Shu YS, Tao W, Miao QB, Zhu YB and Yang YF: Improvement of ventilation-induced lung injury in a rodent model by inhibition of inhibitory KB kinase. J Trauma Acute Care Surg 76: 1417-1424, 2014.

14. Park DW, Jiang S, Liu Y, Siegal GP, Inoki K, Abraham E and Zmijewski JW: GSK3beta-dependent inhibition of AMPK potentiates activation of neutrophils and macrophages and enhances severity of acute lung injury. Am J Physiol Lung Cell Mol Physiol 307: L735-L745, 2014.

15. Bulek K, Liu C, Swaidani S, Wang L, Page RC, Gulen MF, Herjan T, Abbadi A, Qian W, Sun D, et al: The inducible kinase IKKi is required for IL-17-dependent signaling associated with neutrophilia and pulmonary inflammation. Nat Immunol 12: 844-852, 2011.

16. Kravchenko VV, Mathison JC, Schwamborn K, Mercurio F and Ulevitch RJ: IKKi/IKKepsilon plays a key role in integrating signals induced by pro-inflammatory stimuli. J Biol Chem 278: 26612-26619, 2003.

17. Rossi M, Delbauve S, Wespes E, Roumeguere T, Leo O, Flamand V, Le Moine A and Hougardy JM: Dual effect of hemin on renal ischemia-reperfusion injury. Biochem Biophys Res Commun 503: 2820-2825, 2018.

18. Fromowitz FB, Viola MV, Chao S, Oravez S, Mishriki Y, Finkel G, Grimson R and Lundy J: ras p21 expression in the progression of breast cancer. Hum Pathol 18: 1268-1275, 1987.

19. Bonventre JV and Zuk A: Ischemic acute renal failure: An inflammatory disease? Kidney Int 66: 480-485, 2004.

20. Tulafu M, Mitaka C, Hnin Si MK, Abe S, Kitagawa M, Ikeda S, Eishi Y, Kurata S and Tomita M: Atrial natriuretic peptide attenuates kidney-lung crosstalk in kidney injury. J Surg Res 186: 217-225, 2014.

21. White LE, Cui Y, Shelak CM, Lie ML and Hassoun HT: Lung endothelial cell apoptosis during ischemic acute kidney injury. Shock 38: 320-327, 2012.

22. White LE, Santora RJ, Cui Y, Moore FA and Hassoun HT: TNFR1-dependent pulmonary apoptosis during ischemic acute kidney injury. Am J Physiol Lung Cell Mol Physiol 303: L449-L459, 2012.

23. Li XQ, Lv HW, Tan WF, Fang B, Wang $\mathrm{H}$ and Ma H: Role of the TLR4 pathway in blood-spinal cord barrier dysfunction during the bimodal stage after ischemia/reperfusion injury in rats. J Neuroinflammation 11: 62, 2014.

24. Stroo I, Stokman G, Teske GJ, Raven A, Butter LM, Florquin S and Leemans JC: Chemokine expression in renal ischemia/reperfusion injury is most profound during the reparative phase. Int Immunol 22: 433-442, 2010.

25. Smith PD, Puskas F, Meng X, Lee JH, Cleveland JC Jr, Weyant MJ, Fullerton DA and Reece TB: The evolution of chemokine release supports a bimodal mechanism of spinal cord ischemia and reperfusion injury. Circulation 126 (11 Suppl 1): S110-S117, 2012.

26. Aggarwal NR, Tsushima K, Eto Y, Tripathi A, Mandke P, Mock JR, Garibaldi BT, Singer BD, Sidhaye VK, Horton MR, et al: Immunological priming requires regulatory $\mathrm{T}$ cells and IL-10-producing macrophages to accelerate resolution from severe lung inflammation. J Immunol 192: 4453-4464, 2014.

27. Deng J, Wang X, Qian F, Vogel S, Xiao L, Ranjan R, Park H, Karpurapu M, Ye RD, Park GY, et al: Protective role of reactive oxygen species in endotoxin-induced lung inflammation through modulation of IL-10 expression. J Immunol 188: 5734-5740, 2012. 
28. Li M, Khan AM, Maderdrut JL, Simon EE and Batuman V: The effect of PACAP38 on MyD88-mediated signal transduction in ischemia-/hypoxia-induced acute kidney injury. Am J Nephrol 32: 522-532, 2010.

29. Salama M, Farrag SM, Abulasrar SA, Amin MM, Ali AA, Sheashaa H, Sobh M and Arias-Carrion O: Up-regulation of TLR-4 in the brain after ischemic kidney-induced encephalopathy in the rat. CNS Neurol Disord Drug Targets 12: 583-586, 2013.

30. Geng H, WittwerT, Dittrich-Breiholz O, Kracht M and Schmitz ML: Phosphorylation of NF-kappaB p65 at Ser468 controls its COMMD1-dependent ubiquitination and target gene-specific proteasomal elimination. EMBO Rep 10: 381-386, 2009.

31. Moreno R, Sobotzik JM, Schultz C and Schmitz ML: Specification of the NF-kappaB transcriptional response by $\mathrm{p} 65$ phosphorylation and TNF-induced nuclear translocation of IKK epsilon. Nucleic Acids Res 38: 6029-6044, 2010.
32. Fitzgerald KA, McWhirter SM, Faia KL, Rowe DC, Latz E, Golenbock DT, Coyle AJ, Liao SM and Maniatis T: IKKepsilon and TBK1 are essential components of the IRF3 signaling pathway. Nat Immunol 4: 491-496, 2003.

33. Verstrepen L, Verhelst K, Carpentier I and Beyaert R: TAX1BP1, a ubiquitin-binding adaptor protein in innate immunity and beyond. Trends Biochem Sci 36: 347-354, 2011.

This work is licensed under a Creative Commons Attribution-NonCommercial-NoDerivatives 4.0 International (CC BY-NC-ND 4.0) License. 